\title{
Bredsdorff über die Analogie \\ Zur Geschichte des Analogiebegriffs \\ im frühen 19. Jahrhundert
}

Bela Brogyanyi

(Universität Freiburg)

\section{Bredsdorff on Analogy \\ On the History of the "Analogy" Term in the Early $19^{\text {th }}$ Century}

\begin{abstract}
The Danish all-round researcher J. H. Bredsdorff devoted himself to mineralogy and geology, geography, and also to linguistics including runology. In this essay, his booklet Om Aarsagerne til Sprogenes Forandringer (On the Causes of Language Change) from 1821, which passed completely unnoticed at the time of its publishing, will be reexamined, as since the eighties of the nineteenth century it has often been praised as an anticipation of the Neogrammarian theory on analogy. His discourse contains truly interesting approaches to language change, and he also points to speakers' striving for "analogy", i.e. for the regular form. Bredsdorff's concept of analogy can, however, be interpreted only in the sense of the tradition of ancient grammar, and by no means that of Neogrammarian doctrine. In this case, we are thus confronted with a misunderstanding within the history of linguistics. Moreover, Bredsdorff had no influence on the development of linguistic theory in either his own or later periods.
\end{abstract}

\section{Keywords}

J. H. Bredsdorff; reception of ancient grammar; analogy and rule; language change; Neogrammarians

Im Jahr 1821 veröffentlichte der Däne Jakob Hornemann Bredsdorff (1790-1841) die kleine Abhandlung Om Aarsagerne til Sprogenes Forandringer (Über die Ursachen der Sprachveränderungen), in der er viele Fragen des Sprachwandels behandelte, die in die theoretische Diskussion erst viel später Eingang gefunden haben (Pedersen 1962: p. 294, Fn. 3). Die Abhandlung von Bredsdorff (1821) erschien zunächst in einem

1 Vgl. auch Brogyanyi (2016). 
Schulprogramm der Kathedralschule von Roskilde, das als Bredsdorff (1886) in einer Neuauflage von Vilhelm Thomsen, dann als Bredsdorff (1933) in der Ausgabe seiner Abhandlungen zur Sprachwissenschaft und Runologie von Jørgen Glahder erschien. Eine von Uwe Petersen erstellte kommentierte deutsche Übersetzung der kleinen Schrift erschien 1970, eine zweite verbesserte Auflage folgte 1975 (vgl. Bredsdorff 1970 und 1975), die jedoch eher als ein Nachdruck der 1. Auflage als eine 2. verbesserte Auflage zu bezeichnen wäre, wenn wir von der Hinzufügung von drei neuen Literaturangaben auf Seite 50 absehen. Auch eine englische Übersetzung von Henning Andersen liegt vor (Bredsdorff 1982). Andersen fügt seiner Übersetzung auch eine Kritik der deutschen Übersetzung von Uwe Petersen (Bredsdorff 1970 und 1975) an, in der er schreibt:

„The translation by Uwe Petersen (1974 [richtig 1975]) although by and large correct, contains a number of errors and - a more serious criticism, perhaps - deviates from Bredsdorff's text in two ways, in the rendering of the examples and in the treatment of Bredsdorff's transcription." (Bredsdorff 1982: p. 22.)

Dies habe ich selbstverständlich auch bemerkt und neben der deutschen Übersetzung stets den dänischen Originaltext (1821) verwendet.

Um Bredsdorff vorzustellen, möchte ich hier den von R. H. Stamm verfassten Eintrag in SKL (III: p. 900, hier in deutscher Übersetzung) zitieren:

\begin{abstract}
„Bredsdorff, Jacob Hornemann, dänischer Naturforscher und Sprachkundiger (1790-1841), ein Mann mit aussergewöhnlich vielseitigen Interessen, wurde 1814 cand. theol., beschäftigte sich danach mehrere Jahre mit Naturwissenschaften, hielt 1824-26 mineralogische Vorlesungen an der Universität und wurde schliesslich 1828 an der Akademie in Sorø als Lektor für Mineralogie und Botanik angestellt. Aber weder als Wissenschaftler noch als Lehrer konnte er sich auf diesen Gebieten behaupten, und seine diesbezüglichen Schriften sind längst vergessen. Mehr Anerkennung ernteten seine verschiedenen geographischen und historischen Arbeiten." ${ }^{2}$
\end{abstract}

Eine Würdigung von Bredsdorffs Wirken als Sprachforscher bot Otto Jespersen ebenfalls in SKL (III: p. 900, hier in deutscher Übersetzung):

„B[redsdorff] wirkte auch als Sprachforscher, und obwohl seine Arbeiten auf diesem Gebiet weitgefächert waren, erlangten sie in der damaligen Zeit keine grössere Beachtung, man kann sich aber, wenn man sie heute liest, eines gewissen Gefühls von Bewunderung nicht erwehren;

2 Der dänische Originaltext lautet: „Bredsdorff, Jacob Hornemann, dansk Naturforsker og Sprogkyndig (1790-1841), en Mand med ualmindelig alsidige Interesser, blev 1814 cand. theol., beskæftigede sig derpaa fl. Aar med Naturvidenskab, holdt 1824-26 mineralogiske Forelæsninger ved Univ. og ansattes endelig 1828 ved Sorø Akademi som Lektor i Mineralogi og Botanik. Hverken som Videnskabsmand ell. som Lærer formaaede han dog paa disse Omraader at gøre sig gældende, og hans herhen hørende Skr er forlængst glemte. Mere Anerkendelse høstede forsk. geogr. og hist. Arbejder.“ R. H. S[tamm] (SKL III: p. 900). 
er beschäftigt sich nämlich vor allem mit Problemen und Themen, die erst viel später einer eingehenden Behandlung unterzogen wurden, und seine Sichtweisen waren immer vernünftig und natürlich. Mehrere kleine Abhandlungen, besonders der »Versuch einer nach der Aussprache eingerichteten dänischen Rechtschreibung «(1817), beschäftigt sich mit der Lehre über das Hervorbringen der Sprachlaute und mit der sinnvollen Bezeichnung der Laute; als erster gibt er einen zusammenhängenden Vorschlag für eine Lautschrift und hebt die Bedeutung der Lautschrift im Sprachunterricht hervor. Seine Programmschrift (Roskilde 1821) »Über die Ursachen der Sprachveränderungen« zeigt auf wenigen Seiten die Grundzüge einer rationellen Philosophie der Sprachgeschichte; es war sehr bedeutsam, dass Vilh[elm] Thomsen 1886 diese Schrift wieder herausgab, gerade als die Diskussion über dieselben Probleme anlässlich der Theorien der »Junggrammatiker « entbrannte; die Schrift enthält mehr gesunde Vernunft als manche spätere Behandlungen des Themas. Auch als Runologe war B[redsdorff] seiner Zeit voraus (»Ursprung der Runenschrift«, 1822); seine Lesung der Inschrift auf den Goldhörnern ist auch erwähnenswert." ${ }^{3}$

Als Ursachen für die Sprachveränderung führt Bredsdorff in seiner erwähnten Schrift Om Aarsagerne til Sprogenes Forandringer folgende Faktoren an:

1. Fehlerhaftes Hören und Verstehen,

2. Unzuverlässiges Gedächtnis,

3. Unvollkommenheit der Organe,

4. Bequemlichkeit,

5. Streben nach Analogie,

6. Bemühen um Deutlichkeit,

7. Verlangen nach dem Ausdruck neuer Vorstellungen,

8. Einfluss fremder Nationen (d. h. Sprachen).

3 Der dänische Originaltext lautet: „B[redsdorff] virkede ogsaa som Sprogforsker, og om end hans Arbejder, der i denne Retning aldrig var omfattende, ikke i Datiden fik videre Bet., kan man, naar man nutildags læser dem, ikke frigøre sig for en Følelse af Beundring; han beskæftiger sig nemlig væsentlig med Problemer og Emner, der først langt senere blev taget op til indgaaende Behandling, og hans Synsmaader er altid sunde og naturlige. Fl. smaa Afh., især »Prøve af en efter Udtalen indrettet dansk Retskrivning « (1817), beskæftiger sig med Læren om Sproglydenes Frembringelsesmaade og Lydenes rationelle Betegnelse; han er den første til at give en sammenhængede Prøve af Lydskrift og fremhæve Bet. af Lydskrift ved Sprogundervisningen. Hans Programafhandling fra Roskilde $1821 » O m$ Aarsagerne til Sprogenes Forandringer " giver paa faa Sider Grundtrækkene af en rationel Sproghistoriens Filosofi; det var meget $a$ propos, at Vilh[elm] Thomsen 1886 paa ny udgav dette Skrift, netop da Striden om de samme Problemer var stærkt oppe i Anledning af "Junggrammatikernes « (s. d.) Teorier; det indeholder mere sund Fornuft end mange senere Behandlinger af Emnet. Ogsaa som Runolog var B[redsdorff] forud for sin Tid (» Runenskriftens Oprindelse 1822$)$; hans Læsning af Guldhornsindskriften fortjener ogsaa at nævnes. " $O$. Jsp. [Otto Jespersen] (SKL III: p. 900).

Für die Übersetzung der dänischen Originalzitate bin ich Jørn-Ulrik Søndergaard (Silkeborg) zu Dank verpflichtet.

Und ohne die für frühere Autoren übliche Begeisterung lesen wir in Den Store Danske Encyklopædi, III: pp. 343-344 (A. Karl; in deutscher Übersetzung): „Die Schrift Om Aarsagerne til Sprogenes Forandringer (1821) zeigte ihn als einen sprachhistorischen Philosophen." 
Wie die Übersicht von Bredsdorffs Themen zeigt, widmet er der Analogie, d. h. dem Streben nach Analogie, ein gesondertes Kapitel. Bis zur Zeit des Erscheinens dieser Schrift, und sogar bis August Schleichers Aufwertung bzw. Umwertung des Analogiebegriffs in der sprachwissenschaftlichen Forschung (Schleicher 1860: pp. 60ff.), scheint mir eine ähnlich gewichtige Beachtung dieser Erscheinung als Phänomen des Sprachwandels anderswo nicht begegnet zu sein. Bredsdorff ist offenbar der erste, der die Bedeutung dieses Phänomens für die Sprachveränderung mit grosser Klarheit erkannt hat (Thomsen 1927: p. 87, Fn. 1), ganz im Sinne eines Regelwerks, wobei die zwischen zwei konkurrierenden Regeln bestehende Spannung den Wandel bewirkt.

Henning Andersen (1982: pp. 25-26) hat diese Einschätzung in seiner Darstellung bestätigt:

"It may be noted that analogy in Bredsdorff's conception is an immanent characteristic of language, not a force. On one hand, analogy supports the speakers' memory and is a factor for stability... On the other, analogy is what speakers strive to conform to (Stræben efter Analogi) when they deviate from established usage... Bredsdorff recognizes both the interplay of different patterns of analogy and the tension between the propensity for analogy and other causes of change.“

Bredsdorff gebraucht den Terminus Analogie aber nur in der Bedeutung „Regel“ und „Regelmässigkeit“, und in diesem Fall befindet er sich in der grammatischen Tradition seiner Zeit, die auf die antike Grammatik zurückgeht. Dies möchte ich mit einem Zitat von Detlev Fehling erläutern:

„Auf die Frage, was Analogie in der antiken Grammatik der Sache nach bedeutet, geben uns die griechischen Erklärungen eine klare und bündige Antwort: Analogie heißt das, was wir als ‘regelmäßige Flexion' bezeichnen würden. Die Aufgabe des Grammatikers, der sich mit der Analogie befaßt, ist es, die sich in der Flexion gleich verhaltenden Wörter zusammenstellen und die Regel aufzustellen, nach der sie verändert werden.“ (Fehling 1956: pp. 236-237.)

„Doch im allgemeinen wird der Begriff der Analogie sehr viel enger gefaßt und beschränkt sich auf die Regeln, die das System der Deklinationen und Konjugationen begründen, d. h. also die Regeln, die angeben, wie ein im Nominativ gegebenes Substantiv oder ein in der ersten Person sing.praes.ind.act. gegebenes Verb die übrigen Formen bildet, mit anderen Wor-

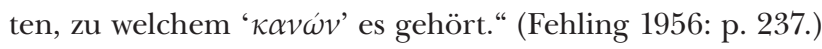

Aber die Bedeutung, die Bredsdorff dieser Erscheinung zuschreibt, ist neuartig, und so kann vielleicht seinen rührigen dänischen Propagatoren doch recht gegeben werden, dass wir es hier tatsächlich mit der „Vorwegnahme späterer Theorien“ zu tun haben (Thomsen 1927: pp. 62-63; Pedersen 1962: p. 294, Fn. 3). Unter „späteren Theorien“ wird die junggrammatische These von den ausnahmslos wirkenden Lautgesetzen und der sie durchkreuzenden Analogie verstanden. Die Analogie der Junggrammatiker voll- 
zieht sich als Prozess auf der Grundlage einer Proportionsformel, abweichende Formen werden nach regelmässigen Formen umgebildet oder durch neugebildete Formen ersetzt. Es handelt sich dabei nach Hermann Paul um „die auflösung einer proportionsgleichung: $a: b-a: x$. Zu drei bekannten grössen wird die vierte unbekannte gefunden.“ (Paul 1880: p. 74; vgl. auch Osthoff \& Brugman 1878: pp. XIII-XIV).

Im Folgenden referiere ich das Kapitel mit dem Titel Streben nach Analogie, welches den Umfang von etwa einer Buchseite hat (Bredsdorff 1821: pp. 16-17; 1886: pp. 22-23; 1970 und 1975: pp. 23-24 und 44-45). Bredsdorffs Begriff „Analogie“ steht eindeutig für die Regelmässigkeit, das „Streben“ könnte man dann als Tendenz zur Regularisierung auffassen.

In dem Kapitel lesen wir in freier Wiedergabe: Am auffallendsten zeigt die Kindersprache die Analogie. Kinder bilden „regelmässige“ Formen, sie sagen eher tiede: tee statt tav 'er schweigt', hvems statt hvis 'wessen', gåse statt ges 'Gänse'. Die Analogie zeigt ihren Einfluss merkbar in jeder Sprache, in der nicht durch die Schrift und andere Mittel die althergebrachten Formen erhalten werden. Der Wirkung der Analogie ist es zuzuschreiben, dass das Dänische heute nur eine Art von Genitivbildung besitzt. So wird der Genitiv heute ohne Abweichung allgemein durch $-s$ in den beiden Genera und den beiden Numeri gebildet (vgl. auch Brøndum-Nielsen 1966: p. III und passim; Jensen 1922: pp. 31-33). Und abweichende Wortformen werden durch regelmässig gebildete ersetzt, so unter dem Einfluss von dä. syg 'krank' dä. sygdom 'Krankheit' statt des älteren sot 'ds.', das heute meist nur in Komposita erscheint, z.B. vatersot 'Wassersucht', svindsot 'Schwindsucht'. Auch auf syntaktischem Gebiet („,i Constructionen“) werden Analogien befolgt, die in früheren Zeiten nicht beachtet worden sind.

Das heisst aber nicht, dass die Sprachen durch Befolgung der Analogie (= Regel) besser oder analoger (= regelmässiger) werden. Das „Streben nach Analogie“ ist nur eine der Tendenzen, die einen sprachlichen Wandel herbeiführen. Es werden aber auch „Unregelmässigkeiten“ (besser: Abweichungen) geschaffen, so dä. øjne ‘Augen’ statt früher øjen zu øje 'Auge'.

Ausser zum Problem der Analogie stellte Bredsdorff auch in Bezug auf die germanische Lautverschiebung scharfsinnige Beobachtungen an (Thomsen 1927: p. 59, Fn. 2; Bredsdorff 1970: p. 31; Pedersen 1962: p. 260, Fn. 1). Dies tat er noch vor Jakob Grimm, jedoch sicherlich unter dem Einfluss von Rasmus Rask, den er aber hier nicht erwähnt hat (Jespersen 1922: pp. 43-44, Fn. 1; Pedersen 1932: pp. XLVIII; vgl. auch Andersen 1982: pp. 27-30).

Vortrefflich sind Bredsdorffs (1821: p. 22, 1970: p. 31) Bemerkungen zum Thema „Wohlklang“, die er wie folgt formuliert hat:

„Man pflegt im allgemeinen den Wohlklang als Grund für einen Teil der sprachlichen Veränderungen anzuführen. ... Die Rücksicht auf den Wohlklang wird ... keine Veränderungen 
in der Sprache verursachen, sondern eher dazu führen, dass man sie unverändert erhält. ... Veränderungen, die allgemein als auf den Wohllaut gegründet angenommen werden, sind zum grössten Teil Folgen der Bequemlichkeit, mitunter auch der Bemühung um Deutlichkeit.“

In einer Zeit, in der das Konzept von Wohlklang oder Wohllaut ganz allgemein den Rang der in späterer Zeit postulierten Lautgesetze besass, sind diese Zeilen höchst interessant. Bredsdorff hat erkannt, dass „Wohlklang“ eben kein linguistischer Begriff ist, wobei diese Vorstellung aber in der Laiensprachbetrachtung bis heute weiterhin aktuell ist.

Bredsdorff blieb für seine Zeitgenossen völlig unbekannt, und so waren seine Leistungen vom forschungsgeschichtlichen Standpunkt aus gesehen irrelevant (Thomsen 1927: p. 63). Es waren später vor allem dänische Sprachwissenschaftler, die sich unermüdlich bemüht haben, ihrem Landsmann gebührende Beachtung zu verschaffen, jedoch mit mässigem Erfolg.

Sievers bespricht in der ersten Auflage seiner Grundzüge der Lautphysiologie im Abschnitt IV kurz auch die Ursachen des Lautwandels (Sievers 1876: pp. 125-129). Aber erst in der 1893 publizierten vierten Auflage wird Bredsdorffs Abhandlung in der Ausgabe von Thomsen (1886) dann in Sievers' Literaturverzeichnis genannt (Sievers 1893: p. 281). Im Text selbst wird sie, soweit ich es überprüfen konnte, allerdings nicht erwähnt. Jespersen (1922: p. 71, Fn. 1) zitiert aus dem Brief eines jungen deutschen Sprachwissenschaftlers (dem er 1886 das Werk von Bredsdorff geschickt hatte), welcher Worte des Staunens über die Aktualität dieser kleinen Arbeit enthält. Leider gibt er den Namen dieses Forschers nicht preis, aber vielleicht handelt es sich doch um Eduard Sievers. Dies wäre zeitlich möglich, da Sievers Bredsdorffs Werk dann in der erwähnten Auflage von 1893 Beachtung schenkt. Delbrück erwähnt Bredsdorff in seinem Aufsatz Das Wesen der Lautgesetze aus dem Jahre 1902 bloss in einer Fussnote, wobei Sievers ihn auf Bredsdorff aufmerksam gemacht hatte (Delbrück 1902: pp. 298, Fn. 1).

Oldřich Hujer schrieb in seinem Nekrolog auf Vilhelm Thomsen (1842-1929):

„In seiner kurzen Geschichte der Sprachwissenschaft (Sprogvidenskapens historie) aus dem Jahr 1902 [siehe die hier in der Übersetzung von Pollak angeführte Ausgabe Thomsen 1927] skizziert er schön die Entwicklung der Sprachwissenschaft bis zu seiner Zeit und bringt einige bis zu der Zeit unbekannte Momente zum Vorschein; so verweist er auf seinen Landsmann J. H. Bredsdorff, der 1790-1841 gelebt hat und ein halbes Jahrhundert seiner Zeit vorausging er hat als erster die Analogie für bestimmte Sprachveränderungen erklärt.“ (Hujer 1927: pp. 171-172; meine Übersetzung aus dem Tschechischen.)

Hujer übernimmt die Begeisterung der dänischen Kollegen, wobei es ihm aber klar gewesen sein dürfte, dass Bredsdorff die Analogie nicht im junggrammatischen, sondern im antiken Sinne verstanden hat.

Der Herausgeber der deutschen Übersetzung schreibt im Kommentar, dass Bredsdorff 
in ausserhalb von Dänemark erschienenen Werken nur von dänischen Autoren (Jespersen 1922 und Thomsen 1927) erwähnt wird (Bredsdorff 1970: p. 37). Wie gezeigt, nimmt ihn Sievers aber spätestens 1893, Delbrück 1902 zur Kenntnis. Aber schon als einer der ersten kommentiert der Amerikaner Benjamin Ide Wheeler Bredsdorffs Schrift, und zwar wohl in Kenntnis der Wiederveröffentlichung von Vilhelm Thomsen (vgl. Bredsdorff 1886), folgendermassen:

„This programm ... published in 1821, and long forgotten, contains in a section on analogy some noteworthy observations fifty years ahead of the times. It exerted, however, no known influence upon the later views concerning language." (Wheeler 1887: p. 44.)

Holger Pedersens dänisch erschienenes Buch über die Geschichte der Sprachwissenschaft im 19. Jahrhundert (Pedersen 1924) wurde in der 1931 erfolgten englischen Übertragung auch im Ausland bekannt (s. Pedersen 1931[1972]). Pedersen erwähnt aber Bredsdorff bloss in zwei Fussnoten, in der ersten im Zusammenhang mit Jacob Grimms germanischer Lautverschiebung, und in der zweiten rühmt er ihn für die Vorwegnahme von Ideen, die erst später in den Siebziger- und Achtzigerjahren des 19. Jahrhunderts in die linguistische Diskussion kamen (vgl. Pedersen 1931[1972]: p. 260, Fn. 1 und p. 294, Fn. 3).

Uwe Petersen wiederholt im Kommentar zu seiner Übersetzung von Bredsdorff bloss kurz die Meinungen der hier behandelten dänischen Autoren. Zum Titel der Abhandlung bemerkt er:

„... daß wir statt von „Ursachen“ dem Gegenstand „Sprache“ noch angemessener mehr von „Bedingungen“, „Voraussetzungen“ (insbes. Kap. 1-5) und schließlich sogar von „Absichten“ und „Zwecken“ (6-7) der Sprachveränderungen sprechen möchten...“ (Bredsdorff 1970: p. 38; 1975: p. 38.)

Der jüngste in der Reihe der Bredsdorff würdigenden Autoren ist Henning Andersen (1982). In seinem Lob übertrifft er alle seine Vorgänger. So sagt er:

„Bredsdorff's contribution to linguistics command admiration for their lucid style, the clear and simple presentation of their arguments, their author's command of data, and - above all - his genius of insight, without which all the other virtues would be of no avail." (Andersen 1982: p. 35.)

Und weiter:

„it would seem that the analysis of Bredsdorff's works and an investigation of the sources of his ideas would be a logical next step in a survey of linguistic thought in early 19th-century Denmark. ... the richness of Bredsdorff's conceptual apparatus and the remarkable clarity and delightful coherence of his ideas entitle him to a central place in such a survey." (Andersen 1982: p. 36.) 
Es handelt sich somit im Fall von Bredsdorff um ein wissenschaftsgeschichtliches Kuriosum; und es ist interessant zu sehen, dass Fragestellungen, die in späteren Zeiten zu stürmischen Auseinandersetzungen geführt haben, oft schon lange vorher reflektiert und in bestimmten Punkten ähnlich behandelt worden sind, jedoch in ihrer Zeit ohne Resonanz geblieben und der Vergessenheit anheimgefallen sind. Thomsen (1927: pp. 62-63) urteilt: „die Schrift ,Om Aarsagerne til Sprogenes Forandringer‘... blieb leider ... ganz unbemerkt und konnte nicht den geringsten Einfluss auf die Entwicklung der Sprachwissenschaft ausüben..."

Es ist aber sicherlich nicht richtig, Bredsdorffs Ideen im Sinne der Junggrammatiker deuten zu wollen, denn seine Bewunderer missinterpretieren seinen Gebrauch des Terminus „Analogie“ völlig. Bredsdorff hat die „Analogie“ als Regel und „das Streben nach Analogie" als Regelausgleich gesehen, wie schon oben gezeigt, und bleibt somit in der Verwendung des Begriffs durchaus der antiken Tradition verhaftet.

Angesichts einer so grossen Begeisterung für einen „Forscher“, dessen Wirken und Leistung erst nach vielen Jahrzehnten entdeckt und propagiert wurden, stellt sich unweigerlich die Frage nach seiner tatsächlichen Bedeutung für die Wissenschaft. Für die Entwicklung der Sprachwissenschaft scheinen die Arbeiten solcher stillen und unbekannten Forscher von untergeordneter Bedeutung zu sein, aber für die Wissenschaftsgeschichte mag es in der Tat interessant sein, die Quellen zu entdecken, aus denen sich die betreffenden Gedankengänge speisten, auch wenn diese Gedankengänge selbst für den direkten Fortgang der Wissenschaft kaum eine Relevanz hatten. Wichtig ist aber die hinter diesen isoliert gebliebenen Ansätzen stehende Geistestradition, die ja auch die Quelle der späteren Hauptströmungen bildet. Somit ist die Aufhellung der Geisteswelt jener Zeit von beachtlicher Bedeutung für die gesamte spätere Forschungsgeschichte.

\section{Bibliographie}

Andersen, H. (1982). Bredsdorff - Life and Work. Historiographia Lingustica, 9, 24-41.

Bredsdorff, J. H. (1821). Om Aarsagerne til Sprogenes Forandringer. Indbydelsesskrift til den offentlige Examen i Roskilde Kathedralskole i September 1821. Kjøbenhavn: Andreas Seidelin.

Bredsdorff, J. H. (1886). Om Aarsagerne til Sprogenes Forandringer (Paa ny udgivet af V. Thomsen). Kjøbenhavn: Gyldendalske Boghandels Forlag.

Bredsdorff, J. H. (1933). J. H. Bredsdorffs Udvalgte Afhandlinger inden for Spragvidenskap og Runologie (Udgivet af J. Glahder). København: Levin \& Munksgaard Forlag.

Bredsdorff, J. H. (1970). Über die Ursachen der Sprachveränderungen (Übersetzt und herausgegeben von U. Petersen). Tübingen: Verlag Gunter Narr.

Bredsdorff, J. H. (1975). Über die Ursachen der Sprachveränderungen (Übersetzt und herausgegeben von U. Petersen; 2. verbesserte Auflage). Tübingen: Verlag Gunter Narr.

Bredsdorff, J. H. (1982). On the causes of linguistic change (1821) (English translation with commentary by Henning Andersen). Historiographia Linguistica, 9, 1-23. 


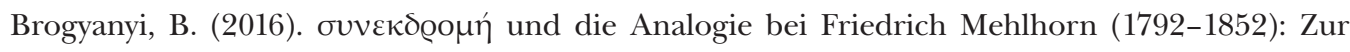
Geschichte des Analogie-Begriffs in der historisch-vergleichenden Sprachwissenschaft. In S. Neri, R. Schuhmann und S. Zeilfelder (Eds.), »dat ih dir it nu bi huldi gibu«. Linguistische, germanistische und indogermanistische Studien Rosemarie Lühr gewidmet. Pp. 73-78. Wiesbaden: Dr. Ludwig Reichert Verlag.

Brøndum-Nielsen, J. (1966). Gammeldansk grammatik. I sproghistorisk fremstilling, III: Substantiverner declination. 2. Oplag. København: J. H. Schultz.

Delbrück, B. (1902). Das Wesen der Lautgesetze. Annalen der Naturphilosophie, 1, 277-308.

Fehling, D. (1956). Varro und die grammatische Lehre von der Analogie und der Flexion. Glotta, 35, 214-270.

Hujer, O. (1927). Drobné zprávy. Listy filologické, 54, 171-172.

Jensen, H. (1922). Neudänische Laut- und Formenlehre. Heidelberg: Carl Winter's Universitätsbuchhandlung.

Jespersen, O. (1922). Language: Its Nature, Development and Origin. London: Allen \& Unwin.

Lund, J. et al. (Eds.). (1995). Den Store Danske Encyklopadi, Tredje bind: Bio - Canberra. København: Gyldendalske Boghandel, Nordisk Forlag.

Pedersen, H. (1924). Sprogvidenskaben i det Nittende Aarhundrede. Metoder og Resultater. København: Gyldendalske Boghandel.

Pedersen, H. (1931 [1962]). The Discovery of Language: Linguistic Science in the Nineteenth Century (Translated by J. W. Spargo). Bloomington-London: Indiana University Press.

Pedersen, H. (1932). Einleitung. In R. K. Rask, Ausgewählte Abhandlungen (Herausgegeben von L. Hjelmslev mit einer Einleitung von H. Pedersen; Vol. I: pp. XIII-LXIII). Kopenhagen: Levin \& Munksgaard.

Sievers, E. (1876). Grundzüge der Lautphysiologie zur Einführung in das Studium der Lautlehre der indogermanischen Sprachen. Leipzig: Breitkopf \& Härtel.

Sievers, E. (1893). Grundzüge der Phonetik zur Einführung in das Studium der Lautlehre der indogermanischen Sprachen (4. vermehrte Auflage). Leipzig: Breitkopf \& Härtel.

SKL = Blangstrup, Ch. et al. (Eds.). (1915). Salmonsens Konversations Leksikon (Vol. III). København: A/S J. H. Schultz Forlagsboghandel.

Thomsen, V. (1927). Geschichte der Sprachwissenschaft bis zum Ausgang des 19. Jahrhunderts (Übersetzt von H. Pollak). Halle a. d. Saale: Max Niemeyer Verlag.

Wheeler, B. I. (1887). Analogy and the Scope of its Application in Language. Ithaca (NY): Wilson.

Bela Brogyanyi / brogpfu@mail.uni-freiburg.de

Sprachwissenschaftliches Seminar

Albert-Ludwigs-Universität Freiburg

Belfortstr. 18, D-79085 Freiburg im Breisgau, Deutschland 
